(2) Open Access Full Text Article

\title{
An open-label pilot study to assess the effectiveness of krill oil with added vitamins and phytonutrients in the relief of symptoms of PMS
}

\section{Michael P Wakeman}

School of Cancer Sciences, University of Birmingham, Edgbaston, Birmingham, West Midlands, UK
Correspondence: Michael P Wakeman University of Birmingham, School of Cancer Sciences, Edgbaston, Birmingham, West Midlands BI5 2TT, UK

Email mikepwakeman@gmail.com
This article was published in the following Dove Press journal:

Nutrition and Dietary Supplements

26 September 2013

Number of times this article has been viewed

\begin{abstract}
An open-label pilot study over 4 months to evaluate the effectiveness of a compound formulation of ingredients, which individually have been demonstrated to be implicated in the pathogenesis of premenstrual syndrome to ameliorate the most troublesome symptoms of the condition. The supplement provided thiamine, riboflavin, pyridoxine, vitamin D, soy isoflavones, rosemary extract, and krill oil and was taken each day for the 3 months of the trial. Statistically significant effect was reported by the 29 women who completed the study in relief of anxiety, bloating, mood swings, breast tenderness, skin outbreaks, food cravings, fatigue, forgetfulness, insomnia, and headache after 3 months of treatment compared with baseline. This pilot study indicates the formulation to be effective, and a larger placebo-controlled trial is now planned.

Keywords: thiamine, riboflavin, pyridoxine, vitamin D, soy isoflavones, rosemary extract, premenstrual syndrome
\end{abstract}

\section{Introduction}

Premenstrual disorders are typically a cluster of somatic and psychological symptoms of varying severity that occur during the luteal phase of the menstrual cycle and resolve during menses. Although premenstrual disorders have been widely recognized for many decades, a precise cause is as yet unknown. Furthermore, there are no definitive, universally accepted diagnostic criteria, and hence differences in the interpretation of significant premenstrual symptoms have led to marked variations in estimated prevalence. ${ }^{1,2}$

PMS (premenstrual syndrome) is a condition that is often poorly understood by patients and their clinicians alike. Indeed, research has only really properly defined the disorder within the past two decades, and as a result only relatively recently have more sophisticated methods to diagnose and treat the condition become apparent. ${ }^{3}$ The diagnosis of PMS is based upon the display of a mixture of psychological and somatic symptoms, which manifest themselves in the luteal phase of the menstrual cycle. ${ }^{4,5}$ The condition is generally managed in primary care; however, it is still debated whether PMS is predominantly psychological or hormonal, and it falls within the remit of gynecologists or psychiatrists. ${ }^{6}$

Symptoms experienced by patients with PMS can be wide-ranging, and many are reported in the literature. ${ }^{7}$ They are generally divided into those that are either predominantly psychological in their nature, predominantly physical, or a combination of both. However, the key discriminator that separates PMS from other disorders is the pattern of symptom presentation. 
Often a PMS patient will experience one or more of the psychological or physical symptoms shown in Table 1 for up to 2 weeks before the commencement of menstruation, at which point they disappear. This is the differentiating pattern which defines PMS. ${ }^{8}$

Hence, PMS is characterized by a cyclical symptom fluctuation, with symptoms appearing premenstrually, abating with the onset of menses, and reappearing again premenstrually in the next cycle. Generally, diagnosis requires marked premenstrual worsening of at least five symptoms that are not exacerbations of other disorders. The Diagnostic and Statistical Manual of Mental Disorders, 4th edition (DSM-IV) indicates that if at least one of the five symptoms is dysphoric, and life activities and relationships are markedly affected, then premenstrual dysphoric disorder (PMDD) should be diagnosed. ${ }^{9}$ A comprehensive review focusing on PMS and other premenstrual disorders was been undertaken in $2008,{ }^{10}$ but according to the definition in the International Classification of Diseases, 10th revision (ICD-10), only one distressing symptom from the following list of physical or behavioral symptoms is required for an ICD-10 diagnosis of PMS according to the definition of Premenstrual tension syndrome N94.3. ${ }^{11}$ Symptoms may include the following:

- Feeling sad, hopeless, or self-deprecating

- Tension, anxiety, mood lability, tearfulness, or persistent irritability

- Anger

- Decreased interest in usual activities

- Difficulty concentrating

- Feeling fatigued

- Changes in appetite

- Hypersomnia or insomnia

- Feeling overwhelmed or out of control

- Other physical symptoms (such as headaches, breast tenderness or swelling, joint or muscle pain, bloating, or weight gain) may be experienced.

Many clinicians consider PMS is distinguished from the normal psychological and somatic premenstrual symptoms

Table I Typical symptoms of PMS

\begin{tabular}{ll}
\hline Psychological & Physical \\
\hline Depression & Breast tenderness/swelling \\
Anxiety & Weight gain \\
Mood swings & Headache \\
Anger/irritability & Joint pain \\
Food cravings & Muscle pain \\
Lethargy & Bloating \\
\hline
\end{tabular}

Abbreviation: PMS, premenstrual syndrome. experienced by many women because of its negative influence on daily functioning and level of distress. However, as seen above, the ICD-10 definition of PMS does not require impact or impairment on daily functioning. This has led to differences in the interpretation of significant premenstrual symptoms and thus to marked variations in the estimated prevalence of the condition. For example, in one study alone, variations range from $80 \%$ to $95 \%$ for physiological premenstrual symptoms, from $30 \%$ to $40 \%$ for PMS, and from $3 \%$ to $8 \%$ for PMDD, ${ }^{12}$ dependent upon interpretation of diagnostic criteria. This is because at one end of the diagnostic spectrum, the ICD-10 criteria are relatively liberal, allowing as few as one or two symptoms with undefined severity to constitute the disorder, whilst at the other extreme, the current American Psychiatric Association Diagnostic (DSM-IV) criteria for premenstrual disorders requires at least $5 / 11$ specific symptoms - including one major mood symptom - and they must occur exclusively during the luteal phase, resolve during menstruation, and be sufficiently severe to interfere with work, family, and social relationships. To reconcile this situation, the International Society for Premenstrual Disorders Expert Consensus Group held its inaugural meeting in September 2008, with the primary aim to provide a unified approach for the diagnostic criteria of premenstrual disorders, their quantification and guidelines on clinical trial design. It is intended that the criteria proposed in that document will inform discussions of the next edition of the World Health Organization's ICD-11. ${ }^{13}$

One of the largest European studies estimated that up to $60 \%$ of women of reproductive age suffer with some symptoms of PMS of varying severity, and 3\%-8\% suffer with the PMDD form of PMS so severe that it causes significant impairment to daily life. Both physical and psychological premenstrual symptoms have significant impact on the quality-of-life as assessed by an ADL (activities of women's daily lives) scoring system, where up to $35 \%$ of women of reproductive age in Europe and Latin America were moderately or severely affected by cyclical premenstrual symptoms. ${ }^{14}$ However, estimates of the prevalence of the condition vary tremendously from country to country. For example, in a study in Switzerland, whilst 91\% of participants reported at least one premenstrual symptom, $10.3 \%$ were diagnosed with PMS, and 3.1\% fulfilled the criteria for PMDD. ${ }^{15}$ In Japan, as many as $95 \%$ of women were found to suffer from premenstrual symptoms, with 5.2\% reporting moderate to severe PMS. ${ }^{16}$ A cross-sectional nationwide survey of a cohort of Spanish women found that of over 2,000 participants, 73.7\% complained of some premenstrual symptoms during the last 
12 menstrual cycles, with $8.9 \%$ reporting moderate/severe PMS and a US population based study reported PMS in $8.3 \%$ of women, whilst a recent study in the People's Republic of China observed a $21.1 \%$ incidence of PMS. ${ }^{17-19}$

Despite the observed variation in incidence, which is undoubtedly influenced by the different interpretation of diagnostic criteria, PMS is found worldwide in diverse cultures, and the World Health Organization has concluded that "a large proportion of women in third world nations in different world regions do experience PMS and data show that it is not a Western Phenomenon." 20

\section{Types and definition of PMS}

The types and definition of PMS are as follows:

- "PMS" - premenstrual symptoms leading up to menstruation and completely relieved by the end of menstruation

○ "Mild" - does not interfere with personal/social and professional life

○ "Moderate" - interferes with personal/social and professional life, but still able to function/interact, although may be suboptimally

○ "Severe" - unable to interact personally/socially and professionally; withdraws from social and professional activities (treatment resistant)

- "Premenstrual exaggeration" - background psychopathology, physical, or other condition, with incomplete relief of symptoms when menstruation ends

- "Premenstrual dysphoric disorder" - a research criterion, not used generally outside the USA. This definition of severe PMS has been adopted by the American Psychiatric Association.

The appearance of symptoms coincides with the luteal phase of the menstrual cycle and occurs after ovulation, when serum progesterone levels rise. Evidence has not shown affected women to have higher or lower levels of progesterone, and it is therefore hypothesized that there is an increased sensitivity to progesterone, which in turn could be attributable to abnormal function or deficiency of neurotransmitters, for example gamma-aminobutyric acid (GABA) or serotonin. ${ }^{21}$ Serotonin plays an important role in other mood disorders; for example, selective serotonin reuptake inhibitors (SSRIs) are used widely in the treatment of depression. Evidence suggests that the serotonin levels or serotonin receptor concentration varies during the menstrual cycle and is under the influence of estrogen and progesterone. Tying in with this theory is the fact that SSRIs improve symptoms in PMS. ${ }^{22}$

\section{Etiology}

The precise etiology of PMS remains undetermined. However, a combination of cyclical ovarian activity together with the effect of estradiol and progesterone on the neurotransmitters serotonin and GABA appear to be key factors. Since PMS is absent before puberty, in pregnancy, and after menopause, this observation supports the theory that cyclical ovarian activity is important. It appears that the rapid fluctuation in estradiol levels, often occurring premenstrually, postnatally, perimenopausally, and sometimes in the same individual can lead to a series of hormone-dependent disorders. Recent research suggests that the risk of PMDD might be associated with the genetic variation in the estrogen receptor alpha gene, but the evidence is still inconclusive. ${ }^{23}$

\section{Management}

The key to effective management of PMS is a correct diagnosis. This needs to be made by the prospective of logging of symptoms by the patient, ideally over two cycles, since retrospective recall is an ineffective tool. Although the approach of reduction of stress is not evidence based, it does appear to help in ameliorating the symptoms, as do dietary interventions such as control of carbohydrate cravings, limitation of alcohol, and reduction of caffeine intake. There are data from nonrandomized trials that show exercise improves PMS symptoms. However, health care practitioners in the UK have available an algorithm for the management of severe PMS from the Royal College of Gynaecologists. $^{24}$

This consists of:

- First line - exercise, cognitive behavior therapy, vitamin B6, combined new generation oral contraceptive pill (cyclically or continuously); continuous or luteal phase (day 15-28) low-dose SSRIs

- Second line - estradiol patches plus oral progesterone; higher dose SSRIs continuously or luteal phase

- Third line-gonadotrophin releasing hormone analogues plus add-back hormone replacement therapy

- Fourth line - total abdominal hysterectomy and bilateral oophorectomy plus hormone replacement therapy (including testosterone).

As a result of these recommendations in the UK, $45 \%$ of prescriptions for PMS are for psychotropic medications, $21 \%$ for hormones, $9 \%$ for vitamins, $9 \%$ for analgesics, and $16 \%$ for other medications. ${ }^{14}$

Hence, there is a justified role, recommendation, and possibly, responsibility to use a natural approach and/or dietary 
interventions as first-line strategies in the management of PMS in order to spare the patient the potential side effects and hazards associated with the latter stages of the step-wise approach outlined above. A number of dietary supplement strategies have been assessed in clinical settings over a number of years and have been found to be beneficial, but few studies have examined the benefits of combining potentially synergistic natural ingredients. Therefore, the formula Cleanmarine ${ }^{\circledR}$ for Women (NOVABRANDS Ltd, Co. Wicklow, Ireland) under investigation represents a new development in terms of bringing the best evidence together in a single presentation, whilst taking into consideration issues such as delivering a therapeutic dose (hence the exclusion of calcium and magnesium) and complying with European legislation regarding potentially active herbs (which explains the absence of herbs such as Vitex agnus-castus extract and Gingko biloba extract, both of which have efficacy data in PMS but which are no longer freely available as a food supplement throughout Europe).

\section{The clinical rationale for Cleanmarine ${ }^{\circledR}$ for Women formula}

\section{Krill oil as a source of omega 3 fatty acids}

The role of long-chain fatty acids for the management PMS has been evaluated in several studies. Omega 3 fatty acids, mainly eicosapentaenoic acid (EPA) and docosahexaenoic acid (DHA), compete with omega 6 species for the enzyme prostaglandin synthetase. The omega 3 fatty acids trigger the secretion of less potent leukotrienes and anti-inflammatory prostaglandins of the 3 series (PGE3, PGI3, and thromboxane A3) and other antiinflammatory compounds such as resolvins and protectins.

Long-chain omega 3 fatty acids in fish oil have been shown to control of cravings prior to menstruation, and omega 3 intake has been demonstrated to be inversely proportional to PMS symptoms. ${ }^{25,26}$ There is significant data positively correlating omega 3 fatty acid levels with brain health, and there are a number of clinical trials identifying a positive effect of dietary supplementation with them in terms of improving depression, anxiety, joint pain, and enhancing mood. ${ }^{27-31}$ There is much debate regarding the manner in which these benefits are effected, but it appears that the supplementation of omega 3 fatty acids helps counterbalance the excess of omega 6 fatty acids which occurs in today's Western diet. Since fatty acids are key components of the lipid raft assembly of all cell membranes within which various receptors reside, it is thought that supplementation with omega 3 fatty acids acts beneficially to favorably affect the functioning of the cell membranes and receptors for various transmitters as well as acting as substrates for antiinflammatory metabolites.

In terms of trials of omega 3 fatty acids specifically for PMS, one randomized, double-blind, controlled trial performed on 184 patients demonstrated that after 45 days from starting the active intervention, the mean severity of depression, anxiety, lack of concentration, and bloating were significantly lower than in the case of the control group. After 90 days of treatment, the mean severity of depression, anxiety, lack of concentration, bloating, nervousness, and the duration of depression, nervousness, anxiety, lack of concentration, bloating, headache, and breast tenderness were significantly improved in the patients receiving active treatment. ${ }^{32}$ A separate trial comparing krill oil with fish oil in PMS identified a significantly enhanced improvement as a result of taking the former over the latter, both after 45 days and 90 days. It also found that women taking krill oil used significantly less painkillers during the period of treatment compared with fish oil. ${ }^{33}$ This superiority may be due to the fact that in krill oil, the beneficial fatty acids (EPA and DHA) are present as phospholipids rather than as triglycerides, as is the case with fish oils. Phospholipids are the form of fatty acid presentation which is preferable for the body, and hence the EPA and DHA present in krill oil are better absorbed and used by the body.

\section{$B$ vitamins}

Thiamine, riboflavin, niacin, and vitamin B6 are required for generating the neurotransmitters potentially involved in the pathophysiology of PMS. In a longitudinal observational study of B vitamin intake and PMS, thiamine and riboflavin intake during the 2-4 years before diagnosis were shown to be inversely associated with incidence of PMS. ${ }^{34}$ Vitamin B6 has been shown to reduce psychological symptoms in PMDD, and a systematic review of nine studies containing 490 patients demonstrated that pyridoxine is effective in relieving overall symptoms of PMS. ${ }^{35}$ A further separate evidence-based review of the role of vitamin B6 in PMS identified seven studies, where there was a significant improvement. ${ }^{36}$

\section{Soy isoflavones}

Sources of natural phytoestrogens such as soya and red clover have been investigated in the management of PMS and found to be of benefit, and soy isoflavone intake has been demonstrated to be inversely proportional to incidence of PMS symptoms. ${ }^{37,38}$ Soy isoflavones have been demonstrated to be beneficial in the relief of menstrual migraine, and 
supplementation has been demonstrated to reduce headache and breast tenderness symptoms in PMS. ${ }^{39,40}$

\section{Rosemary extract}

Rosemary has traditionally been used to elicit an antidepressive effect, and there is exciting new data that demonstrates that this might be effected via modulation of the monoaminergic and cholinergic systems. ${ }^{41}$

\section{Vitamin D3}

Women reporting vitamin D intake of more than $100 \mathrm{IU} /$ day have been shown to have a $70 \%$ lower incidence of PMS after adjusting for age, body mass index, smoking status, and high calcium intake - than those consuming less than this level. Low levels of vitamin D have also been shown to be significantly associated with a higher incidence of mood disorders in PMS. ${ }^{42,43}$

\section{Methods and materials}

Women of reproductive age who fulfilled the diagnostic criteria for PMS were eligible for the study. Women who were pregnant or breastfeeding, on concomitant anticoagulant therapy, receiving psychotherapy, experiencing alcohol or drug dependence, on sex hormones except for oral contraceptives, with a known seafood allergy, or diagnosed with dementia, pituitary disease, or serious medical condition, or taking any prescribed medication for PMS were not eligible for enrolment. Patients taking dietary supplements or vitamins were asked to stop supplementation for 2 weeks prior to the trial, and those patients taking omega 3 fatty acid supplements were excluded from the trial.

Recruitment of potential individuals was undertaken in centers of primary care such as pharmacies. This process consisted of the recruiter providing a cohort of potentially eligible female patients with a written description of the trial and determining their willingness to participate. Upon agreeing to participate, the patient signed a written patient information consent form and was enrolled into the trial. The inclusion procedure established that the women participating had previously been diagnosed with PMS according to ICD-10 criteria; to ensure they were still experiencing typical symptoms of the condition, they completed a confirmatory self-assessment appraisal that they had experienced PMS in the preceding month. Hence, all potential participants were asked to complete a questionnaire that confirmed they had been previously diagnosed with PMS by their general practitioner. In addition, the questionnaire described the typical symptoms of PMS to confirm that the original diagnosis correctly fulfilled the criteria as described above and that the participants were still suffering from these symptoms. The participants completed the questionnaire prior to entry into the trial, and all potential participants met these entry criteria. This confirmation together with data collected during the trial ensured that patients met the diagnostic criteria consistent of PMS in that they had experienced symptoms for 2 months immediately prior to the commencement of the trial. Each patient was then asked to complete a more detailed prospective self-assessment questionnaire over the following month, where participants ranked the typical impact of 10 PMS symptoms - anxiety/irritability, bloating, mood swings, breast tenderness, skin outbreaks, food cravings, fatigue, forgetfulness, insomnia, and headaches - based on a $0-5$ severity rating $(0=$ no symptoms, to $5=$ severe $)$ as well as to record their usual daily consumption of medications. Each patient then answered the same self-assessment questionnaires during each of the next 3 months, when they consumed the krill oil supplement. Following the baseline assessment, each patient was asked to take two of the krill compound supplement capsules each day with meals. The dietary supplement intervention was given for 3 months; all patients were asked not to make other alterations to their usual diet. Because patients currently receiving treatment for PMS were excluded, and since no medical interventions were introduced during the course of the trial - other than the food supplement under investigation - nor any personal data identifying any individuals were collected, the study did not meet the criteria necessitating approval from a UK regional ethics committee. However, the protocol did meet the necessary requirements of the investigators' academic institution. Patients were also provided with access to a helpline, staffed by medically qualified individuals to ensure an adequate duty of care to participants was provided.

A review of the literature performed before the trial commenced revealed that many different approaches have been taken to collect relevant information regarding PMS symptoms in assessment diaries. These vary depending upon the interest of the investigators and the degree of their involvement in data collection, but as yet, there does not appear to be consensus as to the precise tool to be used. ${ }^{44-47}$ The approach taken by this author therefore sought to use best practice observed in similar trials, at the same time being cognizant that the investigation was a pilot open-label study dependent upon patient self-assessment. Hence, the symptom diary was deliberately pragmatic and designed to collect accurate and meaningful data, without being too demanding for participants to complete. Diaries were 
typically completed starting 10 days prior to menstruation, ie, on day 10 following the completion of the previous menstruation and through to day 20. However, since most of the women were generally familiar with the usual pattern of their symptom expression, data collection was empirical rather than prescriptive. The mean age of the participants was $27(18-42)$ and mean body mass index was $23.75 \mathrm{~kg} / \mathrm{m}^{2}$, with a typical regular menstrual bleeding of 5-10 days and menstrual cycles of 25-35 days.

The primary objective of the study was to evaluate the effectiveness of the krill oil formulation for the management of PMS. The study hypothesis was that the krill compound supplement can significantly reduce the physical and emotional symptoms of PMS and be effective in the management of PMS symptoms. Primary outcome measure was based upon the scores of the self-assessment questionnaire for PMS symptoms at baseline compared with those at the end of 3 months, and the efficacy measures were assessed using the paired Student's $t$-test. The study was designed as a prospective, open-label evaluation. Each dose of the krill oil compound supplement contained thiamine hydrochloride $(1.4 \mathrm{mg})$, riboflavin $(1.6 \mathrm{mg})$, pyridoxine hydrochloride (2 mg), standardized soy isoflavones (50 mg; S101), standardized rosemary extract (50 mg; S102) in soft gelatin capsules containing $350 \mathrm{mg}$ of krill oil. Two capsules were taken each day during the active phase of the trial. Patient compliance was monitored by ensuring patients kept a record of their consumption of product, and dispensing a replacement supply of the krill oil supplement only occurred once participants returned their completed diary from the previous month along with their used empty pack of product. All participants completing the trial reported achieving complete compliance.

\section{Results}

A total of 36 PMS sufferers were recruited and completed the baseline questionnaire to assess the impact of PMS on their wellbeing. Twenty-nine records were available for analysis; the remainder were either incomplete or unavailable for a recall.

As illustrated in Table 2, the scores of the self-assessment questionnaire demonstrated a statistically significant difference $(P=0.0001)$ in the reduction of global PMS scores at the end of 3 months treatment with krill oil supplement. The same level of significance was achieved in reduction of anxiety, mood swings, breast tenderness, and food cravings. Highly statistically significant differences were also observed in terms of reducing bloating, skin outbreaks, and forgetfulness, whilst the reduction in terms of severity of fatigue, insomnia, and headache also achieved statistical significance, although not of the same magnitude.

Based upon the global total symptom score at the onset of the trial compared with that of the end of treatment, there was an average $44 \%$ improvement. The improvement for individuals ranged from $10 \%$ to $80 \%$ with no participant failing to report a benefit in global symptom scores. The monthly global total symptom score was calculated by adding together all of the individual recorded symptom scores. The average reductions in symptoms during the same period were as follows: anxiety $38 \%$, bloating $32 \%$, mood $44 \%$, breast tenderness $51 \%$, skin outbreaks $37 \%$, food cravings $37 \%$, fatigue $25 \%$, forgetfulness $35 \%$, insomnia $25 \%$, and headaches $31 \%$. Clearly, some women experienced greater problems with certain specific symptoms than others. Hence, when the individual scores of those participants experiencing the most severe symptoms were examined at onset, and the scores compared with those at the end of the intervention, the following reductions in severity experienced by these specific patients were: anxiety $70 \%$, bloating $69 \%$, breast tenderness $81 \%$, skin outbreaks $69 \%$, cravings $59 \%$, fatigue $60 \%$, forgetfulness $77 \%$, insomnia $62 \%$, and headache $70 \%$.

Only one individual reported an adverse event during the trial. This consisted of "cramping" on the second day of the period during month 1 of the trial, but this was resolved within a day and it is unknown whether this was related to consumption of the krill oil supplement. In contrast, other patients reported unexpected ad-hoc benefits such as "stiff joints improved," "hair shinier," "nails improved," "used to suffer with dry eyes, but this has cleared up," and "energy levels have increased."

\section{Discussion}

PMS was first described in 1931 by Frank and Horney who speculated on the possible pathophysiological origins of the condition and on some forms of treatment. ${ }^{48,49}$ A good working definition of PMS is: a condition which manifests with distressing physical, behavioral, and psychological symptoms not due to organic or underlying psychiatric disease, which regularly occurs during the luteal phase of each menstrual (ovarian) cycle, which disappears, or significantly regresses by the end of menstruation. The severity of symptoms is judged according to the degree of interference with day-today activities and relationships.

Today it is well recognized that many women experience mild physical and emotional PMS symptoms, which are 
Table 2 Mean patient score for recorded symptoms at baseline and after 3 months of treatment

\begin{tabular}{|c|c|c|c|c|c|}
\hline \multirow[t]{2}{*}{ Symptom } & \multicolumn{2}{|c|}{ Baseline } & \multicolumn{2}{|c|}{ After 3 months } & \multirow[t]{2}{*}{$P$-value ${ }^{a}$} \\
\hline & $\begin{array}{l}\text { Mean } \\
\text { score }\end{array}$ & SD & $\begin{array}{l}\text { Mean } \\
\text { score }\end{array}$ & SD & \\
\hline \multicolumn{6}{|l|}{$n=29$} \\
\hline Anxiety & 3.24 & 1.09 & 2 & 0.8 & 0.0001 \\
\hline Bloating & 2.93 & 1.25 & 1.97 & 0.78 & 0.0008 \\
\hline Mood swings & 3.45 & 1.18 & 1.9 & 0.98 & 0.0001 \\
\hline Breast tenderness & 2.69 & 1.47 & 1.31 & 0.89 & $0.000 \mathrm{I}$ \\
\hline Skin outbreaks & 2.34 & 1.37 & $\mathrm{I} .48$ & 0.74 & 0.0016 \\
\hline Food cravings & 3.03 & 1.09 & 1.9 & 1.11 & 0.0001 \\
\hline Fatigue & 2.83 & 1.17 & 2.17 & 0.76 & 0.02 \\
\hline Forgetfulness & 2.31 & I.II & 1.48 & 1.02 & 0.008 \\
\hline Insomnia & 1.83 & 1.31 & 1.38 & 1.12 & 0.05 \\
\hline Headache & 1.79 & 1.52 & 1.24 & 1.06 & 0.03 \\
\hline Global & 26.59 & 6.06 & 16.79 & 5.72 & 0.0001 \\
\hline
\end{tabular}

Note: a Calculated using paired student's $t$-test.

Abbreviation: SD, standard deviation.

not particularly troublesome. However, when more severe, these symptoms can impact on day-to-day life, interfere with normal functioning, and even lead to a breakdown in interpersonal relationships. Given the impact of the condition upon those who experience it and the potential side effects and hazards associated with conventional medical interventions, most clinicians would agree that investigating the appropriateness and benefits of natural approaches represents a meaningful first potential intervention worthy of investigation. There is good evidence to suggest that certain complementary and alternative medicine approaches such as cognitive behavioral therapy and nutritional interventions using supplements can be of benefit in certain patients.

\section{Conclusion}

There is significant data to suggest that the dietary supplement ingredients which form the basis of the active components of the formulation investigated in this trial each individually have a beneficial effect in relieving different symptoms of PMS. This open-label pilot study investigated the likelihood that combining these ingredients together might synergistically deliver these benefits in a single-dose presentation.

It is acknowledged that keeping a record of symptoms can in itself contribute a healing effect. To attempt to diminish this effect, an assessment of severity of each symptom under evaluation was made for the month prior to entry into the trial and then the same data was collected for another month without treatment. A review of symptom scores at month 1 of active treatment compared with baseline data did not demonstrate a significant improvement in symptoms which would suggest the effect of recording symptoms made little contribution to the outcome, although this effect cannot be completely dismissed. However, the delay in response observed here is consistent with other trials using krill oil in PMS where benefit maximally accrued at 90 days and typical of nutritional interventions featuring essential fatty acid supplements which often take 2-3 months to achieve a positive outcome. ${ }^{50}$

The findings of this study reflect those of other authors who have identified omega 3 polyunsaturated fatty acids as a therapeutic option for PMS along with those studies which have shown the efficacy of nutrients such as B vitamins, soy isoflavones, and rosemary in relieving likely individual PMS symptoms.

A statistically significant improvement in all symptoms which were assessed was achieved in this open-label pilot study, which by its very nature was limited in terms of patient numbers and controlled elimination of possible confounders due to the self-assessment nature of the data collection. The trial was limited by the fact that it did not include a comparison group and was neither placebo-controlled nor double-blinded. Furthermore, the nature of the condition and the high level of placebo response reported in some trials also need to be taken into consideration in the interpretation of the positive outcome. Nevertheless, the results suggest Cleanmarine ${ }^{\mathbb{R}}$ for Women represents a likely safe and effective first stage intervention for PMS and is worthy of further investigation in this condition in a double-blind placebo-controlled setting using a validated instrument to collect symptom data.

\section{Disclosure}

The author reports no conflicts of interest in this work.

\section{References}

1. Freeman EW, Sondheimer SJ. Premenstrual dysphoric disorder: Recognition and treatment. Prim Care Companion J Clin Psychiatry. 2003;5:30-39

2. Halbreich U. The diagnosis of premenstrual syndromes and premenstrual dysphoric disorder-clinical procedures and research prospectives. Gynaecol Endocrinol. 2004;19:320-334.

3. Steiner M, Streiner DL, Steinberg S, et al. The measurement of premenstrual mood symptoms. J Affect Disord. 1999;53:269-273.

4. Freeman EW, Sondheimer SJ. Premenstrual dysphoric disorder: recognition and treatment. Prim Care Companion J Clin Psychiatry. 2003;5:30-39.

5. Halbreich U. The diagnosis of premenstrual syndromes and premenstrual dysphoric disorder - clinical procedures and research prospectives. Gynecol Endocrinol. 2004;19:320-334.

6. Baker LJ, O’Brien PMS. Premenstrual syndrome (PMS): a perimenopausal perspective. Maturitas. 2012;72:121-125.

7. Dennerstein L, Lehert P, Keung LS, Pal SA, Choi D. A populationbased survey of Asian women's experience of premenstrual symptoms. Menopause Int. 2010;16:139-145.

8. O’Brien PMS, Bäckström T, Brown C, et al. Towards a consensus on diagnostic criteria, measurement and trial design of the premenstrual disorders: the ISPMD Montreal consensus. Arch Womens Ment Health. 2011;14;13-21. 
9. American Psychiatric Association. Diagnostic and Statistical Manual of Mental Disorders. 4th ed. Washington DC: American Psychiatric Association; 1994.

10. Halbreich U, Backstrom T, Eriksson et al. Clinical diagnostic criteria for premenstrual syndrome and guidelines for their quantification for research studies. Gynaecol Endocrinol. 2007;23:123-130.

11. World Health Organization (WHO). International Statistical Classification of Diseases and Related Health Problems, 10th revision. N94.3 Premenstrual Tension syndrome. 2nd ed. Geneva: WHO; 2007.

12. Pearlstein T. Prevalence, impact, on morbidity and burdern of disease. In: O'Brien PMS, Rapkin A, Schmidt P, editors. The Premenstrual Syndromes: PMS and PMDD. London: Informa Healthcare; 2007:37-47.

13. O'Brien PMS, Backstrom T, Brown C, et al. Towards a consensus on diagnostic criteria, measurement and trial design of premenstrual disorders: the ISPMD Montreal consensus. Arch Womens Mental Health. 2011;14:13-21.

14. Weisz G, Knaapen L. Diagnosing and treating premenstrual syndrome in five western nations. Social Sci Med. 2009;68:1498-1505.

15. Tschuidin S, Bertea PC, Zemp E. Prevalence and predictors of premenstrual syndrome and premenstrual dysphoric disorder in a population based sample. Arch Womens Mental Health. 2010;13:485-494.

16. Takeda T, Tasaka K, Sakata M, Murata Y. Prevalence of premenstrual syndrome and premenstrual dysphoric disorder in Japanese women. Arch Womens Mental Health. 2006;9:209-212.

17. Duenas Jl, Lete I, Bermejo, et al. Prevalence of premenstrual syndrome and premenstrual dysphoric disorder in a representative cohort of Spanish women of fertile age. Eur J Obstet Gynaecol Reprod Biol. 2011;156:72-77.

18. Duester PA, Adera T, South-Paul J. Biological, social and behavioural factors associated with premenstrual syndrome. Arch Fam Med. 1999;8:122-128.

19. Qiao M, Zhang H, Liu H, et al. Prevalence of premenstrual syndrome and premenstrual dysphoric disorder in a population based sample in China. Eur J Obstet Gynaecol Reprod Biol. 2012;162:83-86.

20. Ericksen KP. Menstrual symptoms and menstrual beliefs: national and cross national patterns. In: Ginsberg BE, Carter BF, editors. PMS: Ethical and Legal Implications in a Biomedical Perspective. New York: Plenium; 1987:175-188.

21. Yonkers KA, O’Brien PMS, Eriksson E. Premenstrual syndrome. Lancet. 2008;371;1200-1210.

22. Rubinow DR, Hoban MC, Grover GN, et al. Changes in plasma hormones across the menstrual cycle in patients with menstrually related mood disorder and in control subjects. Am J Obstet Gynecol. 1988;158:5-11.

23. Huo L, Straub RE, Roca C, et al. Risk for premenstrual dysphoric disorder is associated with genetic variation ESR1, the estrogen receptor alpha gene. Biol Psychiatry. 2007;62:925-933.

24. Royal College of Obstetricians and Gynaecologists. Premenstrual Syndrome, Management (Green-top 48). London: Royal College of Obstetricians and Gynaecologists; 2007. Available from: http://www. rcog.org.uk/files/rcog-corp/uploaded-files/GT48ManagementPremens turalSyndrome.pdf. Accessed August 26, 2013.

25. Horrobin DF. The role of essential fatty acids and prostaglandins in the premenstrual syndrome. J Reprod Med. 1983;28:465-468.

26. Giugliano D, Torella R. Prostaglandin E1 inhibits glucose-induced insulin secretion in man. Prostaglandins Med. 1978;1:165-166.

27. Wyatt KM, Dimmock PW, Frischer M, et al. Prescribing patterns in premenstrual syndrome. BMC Womens Health. 2002;2(1):4.

28. Barnes J, Ernst E. Traditional herbalists' prescriptions for common clinical conditions: a survey of members of the UK National Institute of Medical Herbalists. Phytother Res. 1998;12:369-371.

29. Deutch B. Menstrual pain in Danish women correlated with low n-3 polyunsaturated fatty acid intake. Eur J Clin Nutr. 1995;49:508-516.
30. Deutch B. Painful menstruation and low intake of n-3 fatty acids. Ugeskr Laeger. 1996;158:4195-4198. Danish.

31. Harel Z, Biro FM, Kottenhahn RK, Rosenthal SL. Supplementation with omega-3 polyunsaturated fatty acids in the management of dysmenorrhea in adolescents. Am J Obstet Gynecol. 1996;174:1335-1338.

32. Sampalis F, Bunea R, Pelland MF, et al. Evaluation of the effects of Neptune Krill Oil on the management of premenstrual syndrome and dysmenorrhea. Altern Med Rev. 2033;8:171-179.

33. Sohrabia N, Kashaniana M, Ghafooria SS, et al. Evaluation of the effect of omega-3 fatty acids in the treatment of premenstrual syndrome: "a pilot trial". Complement Ther Med. 2013;21;141-146.

34. Chocano_bedoya P, Manson JE, Hankinson SE, et al. Dietary B vitamin intake and incident premenstrual syndrome. Am J Clin Nutr. 2011;93: 1080-1086.

35. Panay N. Management of premenstrual syndrome: evidence-based guidelines. Obstet Gynecol Reprod Med. 2011;21:221-228.

36. Dennehy CE. The use of herbs and dietary supplements in gynecology: an evidence based review. J Midwifery Womens Health. 2006;51: 402-406.

37. Kim HW, Kwon MK, Kim NS, Reame NE. Intake of dietary soy isoflavones in relation to perimenstrual symptoms of Korean women living in the USA. Nurs Health Sci. 2006;8:108-113.

38. Cassidy A, Bingham S, Setchell KD. Biological effects of a diet of soy protein rich in isoflavones on the menstrual cycle of premenopausal women. Am J Clin Nutr. 1994;60:333-340.

39. Bryant M, Cassidy A, Hill C, et al. Effect of consumption of soy isoflavones on behavioural, somatic and affective symptoms in women with premenstrual syndrome. Br J Nutr. 2005;93:731-739.

40. Taher S, Cahill A, Eliahoo J, et al. Randomised placebo controlled pilot study on comparing isoflavones versus placebo for the treatment of premenstrual syndrome. Maturitas. 2009;63:S114-S114.

41. Machado DG, Bettio LEB, Cuhna MP, et al. Antidepressant-like effect of the extract of rosemarinus officinalis in mice: involvement of the monoaminergic system. Prog Neuro-Psychopharmacol Biol Psychiatry. 2009;33:642-650

42. Murphy PK, Wagner CL. Vitamin D and mood disorders among women: an integrative review. J Midwifery Womens Health. 2008;53: 440-446.

43. Bertone-Johnson ER, Chocano-Bedoya PO, Zagarins SE, Micka AE, Ronnenberg AG. Dietary vitamin D3 levels and premenstrual syndrome in a college-aged population. J Steroid Biochem Mol Biol. 2010;121: 434-437.

44. Epperson CN, Amin Z, Mason GF. Pathophysiology II: neuroimaging, GABA and the menstrual cycle. In: O'Brien PMS, Rapkin A, Schmidt P, editors. The Premenstrual Syndromes: PMS and PMDD. London: Informa Healthcare; 2007:99-107.

45. Bastani F, Hashemi S. Effects of a web based lifestyle education on general health and severity of symptoms of premenstrual syndrome among female students: a randomised controlled trial. Social Behav Sci. 2012;46:3356-3362.

46. Steiner M, Peer M, Macdougall M, Hasket R. The premenstrual tension syndrome rating scales: an updated version. J Affect Disord. 2011;135: 82-88.

47. Budeiri DJ, Li Wan Po A, Dornan JC. Clinical trials of treatments of premenstrual syndrome: entry criteria and scales for measuring outcomes. Br J Obstet Gynaecol. 1994;101:689-695.

48. Horney K. Die prämenstruellen Verstimmungen. [The Premenstrual upset]. Zeitschrift für Psychoanalytische Pädagogik. 1931;5:1-7.

49. Frank RT. The hormonal basis of premenstrual tension. Arch Neurol Psychiatry. 1931;26:1053-1057.

50. Horrobin DF, Manku MS. Premenstrual syndrome and premenstrual breast pain (cyclical mastalgia): disorders of essential fatty acid (EFA) metabolism. Prostaglandins Leukot Essent Fatty Acids. 1989;37; 255-261. 
Nutrition and Dietary Supplements

Dovepress

\section{Publish your work in this journal}

Nutrition and Dietary Supplements is an international, peer-reviewed, open access journal focusing on research into nutritional requirements in health and disease, impact on metabolism and the identification and optimal use of dietary strategies and supplements necessary for normal growth and development. The journal welcomes papers covering original research, basic science, clinical \& epidemiological studies, reviews and evaluations, guidelines, expert opinion and commentary, case reports and extended reports. The manuscript management system is completely online and includes a very quick and fair peer-review system, which is all easy to use.

Submit your manuscript here: http://www.dovepress.com/nutrition-and-dietary-supplements-journal 